\section{Nível de atividade física de coletores de lixo de duas cidades de porte médio do sul do Brasil}

\author{
Physical activity level of garbage collectors \\ from two medium-sized cities in southern \\ Brazil
}

Rodrigo Kohn Cardoso ${ }^{1}$

Airton José Rombaldi i,2

Marcelo Cozzensa da Silva ${ }^{1,2}$

\section{Resumo}

O objetivo do estudo foi verificar os níveis de atividade física (AF) total e nos diferentes domínios de coletores de lixo. Este estudo caracterizou-se como observacional descritivo e do tipo censo. A população foi composta por todos os coletores de lixo das cidades de Pelotas e Rio Grande, Rio Grande do Sul. A coleta de dados foi realizada por meio de um questionário contendo questões relativas a variáveis sociodemográficas, econômica, nutricional e comportamentais. O nível de atividade física foi avaliado através do Questionário Internacional de Atividade Física, IPAQ - versão longa. Foi realizada a análise univariada de todas as variáveis coletadas, com cálculo das medidas de tendência central e dispersão para as variáveis contínuas e proporções para as variáveis categóricas. Foram entrevistados todos os 127 coletores de lixo, sendo que a média de AF total relatada pelos trabalhadores foi de 4292,0 minutos por semana (DP 1034,2). Quando verificada a AF ocupacional, o escore médio encontrado foi de 3853,0 minutos por semana (DP 583,4). Os demais domínios, deslocamento, lazer e doméstico, apresentaram médias de AF semanal respectivamente de, 257,9 (DP 237,5), 77,6 (DP 111,5) e 103,5 (DP $101,8)$ minutos. Concluiu-se que os coletores de lixo estavam expostos a uma carga de AF total e ocupacional extremamente elevadas, podendo haver prejuízo significativo para a saúde se não forem adotadas medidas adequadas de nutrição e recuperação física.

\section{Palavras-chave}

Epidemiologia, Trabalhadores, Saúde ocupacional, Lixo.

\begin{abstract}
The aim of the present study was to verify levels of total physical activity $(P A)$ and $P A$ in different domains of garbage collectors. This study was characterized as descriptive and observational type Census. The population consisted of all the garbage collectors of Pelotas and Rio Grande cities, Southern Brazil. Data collection was conducted through a questionnaire with sociodemographic, economic, nutritional and behavioral questions. The level of PA was evaluated by the International Physical Activity Questionnaire, IPAQ - long version. It was performed univariate analysis of all variables collected, calculating measures of central tendency and dispersion for continuous variables and proportions for categorical variables. We interviewed all 127 garbage collectors, and the mean of total $P A$ reported was 4292.0 (SD 1034.2) minutes per week. When verified occupational PA, the mean score found was 3853.0 (SD 583.4) minutes per week. The remaining domains, commuting, leisure and domestic had, respectively, means of 257.9 (SD 237.5), 77.6 (SD 111.5) and 103.5 (SD 101.8) minutes per week. It was concluded that garbage collectors were exposed to extremely high levels of total and occupational $P A$ which may cause significant impairment to health if not taken adequate measures of nutrition and physical recovery.
\end{abstract}

\section{Keywords}

Epidemiology, Workers, Occupational health, Garbage.
Rev Bras Ativ Fis Saúde p. 604-613 DOI:

http://dx.doi.org/10.12820/rbafs.v.18n5p604

1 Programa de Pós graduação em Educação Física / Universidade Federal de Pelotas

2 Grupo de Estudos em Epidemiologia da Atividade Física (GEEAF) 


\section{INTRODUÇÃO}

A crescente ampliação das áreas urbanas tem contribuído para o crescimento de impactos ambientais negativos, dentre os quais se destaca a produção de lixo. Em 2010, o Brasil produziu 195 mil toneladas de resíduos sólidos por dia, um aumento de $6,8 \%$ em relação a $2009^{1}$. Neste contexto, o serviço de limpeza urbana, caracterizado principalmente pela coleta de lixo, é extremamente importante para a preservação ambiental e para a saúde da comunidade ${ }^{2}$.

A execução da tarefa de coleta de lixo pode ser feita de forma manual, semiautomatizada ou automatizada, variando de acordo com o desenvolvimento tecnológico do local, características demográficas da região e nível educacional e cultural da população ${ }^{2}$. Contudo, esta atividade está entre as ocupações de maior risco para a saúde dos trabalhadores ${ }^{3}$.

Os coletores de lixo estão constantemente expostos a esforços físicos durante a realização de suas atividades laborais, os quais envolvem caminhadas, corridas, subidas e descidas de ladeiras e levantamento e transporte de pesos variados ao longo do dia $^{4,5}$. Um estudo realizado por Anjos e colaboradores ${ }^{6}$, concluiu que o gasto energético de um coletor de lixo durante o trabalho variou entre 288,4 e 319,1 kcal por hora, podendo alcançar um gasto de até $2000 \mathrm{kcal}$ por período de trabalho, caracterizando o trabalho como pesado, de acordo com a Organização Mundial de Saúde.

É sabido que a realização de atividades físicas (AF) regulares auxilia na melhora e manutenção da saúde através da prevenção de diversas doenças crônicas não transmissíveis e na mortalidade prematura ${ }^{7}$. A prática regular de $\mathrm{AF}$, como caminhadas, pedaladas, participação em atividades esportivas e atividades ocupacionais proporciona benefícios importantes para a saúde ${ }^{7}$, no entanto, a realização exagerada e sem os cuidados necessários, pode torná-la prejudicial.

Considerando a preocupação com a saúde e qualidade de vida dos trabalhadores e a importância da tarefa que realizam, é de fundamental importância se verificar os níveis de AF dos coletores de lixo. Apesar de alguns estudos determinarem a exigência física e ergonômica desses trabalhadores ${ }^{5,6}$, ainda são escassas as pesquisas que avaliam esse tema específico. Portanto, o objetivo do presente estudo foi determinar o nível de atividade física (AF) total e nos diferentes domínios (lazer, deslocamento, ocupacional e doméstico) dos coletores de lixo de duas cidades de porte médio do Rio Grande do Sul/Brasil.

\section{MÉTOdOS}

A pesquisa foi realizada nas cidades de Pelotas e Rio Grande, ambas localizadas no extremo sul do Brasil. Pelotas é uma cidade com 328.864 habitantes e, de acordo com o Serviço Autônomo de Saneamento de Pelotas são recolhidos cerca de 160 toneladas de lixo por dia na cidade. A cidade de Rio Grande possui 198.048 habitantes e produz cerca de 130 toneladas de lixo por dia, de acordo com a Secretaria Municipal de Serviços Urbanos de Rio Grande.

O estudo se caracterizou como sendo observacional descritivo do tipo censo. A população foi composta por todos os coletores de lixo da zona urbana das cidades de Pelotas e Rio Grande. Todos os coletores de lixo das duas cidades, estando ativos ou não (licença médica ou férias), foram incluídos no estudo. Os motoristas foram excluídos do estudo.

As entrevistas foram realizadas por oito entrevistadores previamente treinados os quais estavam cursando o ensino superior. $\mathrm{O}$ treinamento foi aplicado pelo pes- 
quisador responsável e teve duração de doze horas, onde foram abordadas questões referentes a técnicas de entrevistas, conhecimento do questionário e simulação da entrevista. Foi realizado um estudo piloto com coletores de lixo de uma cidade vizinha as estudadas como parte do treinamento dos entrevistadores e verificação da compreensão das questões que compunham o questionário.

As informações foram coletadas através de um questionário pré-codificado com questões fechadas. Foi realizado o controle de qualidade que consistiu na aplicação de um questionário com questões-chave a 5\% dos entrevistados.

O questionário utilizado na coleta de dados continha questões medindo as seguintes características: econômicas - nível econômico (de acordo com o instrumento da Associação Brasileira de Empresas de Pesquisa - ABEP- classe A mais elevada) ${ }^{8}$ e renda familiar (em reais); sociodemográficas - idade (coletada em anos completos), cor da pele (observada pelo entrevistador); comportamentais - tabagismo (número de cigarros fumados nos últimos 30 dias. Respondentes foram classificados em fumante, ex-fumante, não fumante) e ingestão de bebidas alcoólicas (através do instrumento Cut down, Annoyed by criticism, Guilty and Eye-opener - CAGE) ${ }^{9}$; Nutricional - índice de massa corporal (IMC) (medido pelo peso (kg) referido, dividido pela estatura $(\mathrm{cm})$ referida elevada ao quadrado); características do trabalho - tempo de trabalho (em meses) e período de trabalho diário (horas trabalhadas/dia). A principal variável de interesse, o nível de AF nos quatro domínios - trabalho, lazer, doméstico e deslocamento - foi avaliada utilizando-se a versão longa do International Physical Activity Questionnaire (IPAQ2 ${ }^{10}$, instrumento validado em diversos países, incluindo países da América Latina ${ }^{10}$. A versão longa estimou, separadamente, o nível de atividade física nos contextos do trabalho, lazer, domiciliar e no deslocamento, além de permitir o cálculo do escore total despendido nas atividades. $\mathrm{O}$ mesmo foi aplicado face-a-face e as perguntas foram referentes a quantidade de AF realizadas em uma semana habitual.

A coleta de dados foi realizada no período de 4 de dezembro de 2011 e 14 de janeiro de 2012. Os entrevistadores deslocaram-se em equipes para as sedes da empresa, onde realizaram a aplicação dos questionários. A aplicação das entrevistas teve duração média de 30 minutos e foi acompanhada de supervisão durante todo o trabalho de campo.

Os questionários foram duplamente digitados no programa EPIDATA 3.1 e o pacote estatístico STATA 10.0 foi utilizado para a realização das análises. Foi realizada a análise univariada de todas as informações coletadas, com cálculo das medidas de tendência central (mediana, média, desvio padrão [DP] e valores mínimo e máximo) para as variáveis contínuas e de proporções para as variáveis categóricas.

O protocolo do estudo foi submetido e aprovado pelo Comitê de Ética em Pesquisa da Escola Superior de Educação Física da Universidade Federal de Pelotas sob número 003/2011. Os princípios éticos foram assegurados às empresas e aos funcionários, de modo que todos consentiram em participar do estudo.

\section{RESULTADOS}

Foram entrevistados todos os 127 coletores de lixo (100\% da população) vinculados à empresa responsável pela coleta de lixo nas cidades de Rio Grande e Pelotas, incluindo os cinco indivíduos que encontravam-se de licença médica durante o período de coleta de dados. Todos os indivíduos entrevistados eram do sexo masculino, sendo que 76 trabalhavam na cidade de Pelotas (59,8\%). A média de idade da população em estudo foi de 26,2 anos (DP 5,4), 57,5\% eram de cor da pele 
branca e 70,1\% eram casados ou viviam com companheira. O tempo médio de escolaridade foi de 6,2 anos (DP 2,3), e o salário médio dos trabalhadores foi de R $\$$ 834,40 reais (DP 159,6), incluindo o valor recebido nas horas extras trabalhadas (Tabela 1).

Tabela 1 - Prevalência de variáveis sociodemográficas, nutricional, comportamentais e características do trabalho dos coletores de lixo das cidades de Pelotas e Rio Grande/RS, 2012 ( $n=127)$.

\begin{tabular}{|c|c|}
\hline VARIÁVEIS & $\begin{array}{c}\text { PREVALÊNCIA } \\
\mathrm{N}(\%)\end{array}$ \\
\hline \multicolumn{2}{|l|}{ Idade } \\
\hline $18-21$ & $26(20,5)$ \\
\hline $22-25$ & $39(30,7)$ \\
\hline $26-29$ & $29(22,8)$ \\
\hline 30 ou mais & $33(26,0)$ \\
\hline \multicolumn{2}{|l|}{ Cor da pele } \\
\hline Branca & $54(42,5)$ \\
\hline Não branca & $73(57,5)$ \\
\hline \multicolumn{2}{|l|}{ Escolaridade } \\
\hline Fundamental incompleto & $31(24,4)$ \\
\hline Fundamental completo & $56(44,1)$ \\
\hline Médio & $38(29,9)$ \\
\hline Superior incompleto & $2(1,6)$ \\
\hline \multicolumn{2}{|l|}{ Situação conjugal } \\
\hline Casado ou com companheira & $89(70,1)$ \\
\hline Solteiro ou sem companheira & $33(26,0)$ \\
\hline Separado & $5(3,9)$ \\
\hline \multicolumn{2}{|l|}{ Tabagismo } \\
\hline Nunca fumou & $66(52,0)$ \\
\hline Ex-fumante & $18(14,0)$ \\
\hline Fumante & $43(34,0)$ \\
\hline \multicolumn{2}{|c|}{ Índice de Massa Corporal (kg/m²) } \\
\hline Normal & $103(81,7)$ \\
\hline Sobrepeso / Obesidade & $23(18,3)$ \\
\hline \multicolumn{2}{|l|}{ Tempo de trabalho (meses) } \\
\hline Menos de 1 & $22(17,3)$ \\
\hline $1-12$ & $49(38,6)$ \\
\hline $13-60$ & $38(29,9)$ \\
\hline Mais de 60 & $18(14,2)$ \\
\hline \multicolumn{2}{|l|}{ Alimentação (refeições/dia) } \\
\hline $0-3$ & $52(40,9)$ \\
\hline $4-5$ & $69(54,3)$ \\
\hline 6 ou mais & $6(4,7)$ \\
\hline \multicolumn{2}{|c|}{ Indicativo para alcoolismo (CAGE) } \\
\hline Negativo & $107(84,9)$ \\
\hline Positivo & $19(15,1)$ \\
\hline
\end{tabular}

Mais da metade dos coletores de lixo nunca havia fumado (52,0\%), 15,1\% apresentou indicativo para alcoolismo e mais de 4/5 dos trabalhadores esteve na categoria normal de acordo com a classificação de IMC. Quanto ao tempo de trabalho, os coletores apresentaram uma mediana de nove meses de atividade laboral, tendo zero e 240 meses como períodos mínimo e máximo respectivamente. A mediana de carga horária diária de trabalho foi de oito horas/dia, tendo seis e quatorze horas/dia como tempo mínimo e máximo, respectivamente. A tabela 1 apresenta a descrição sociodemográfica, nutricional, comportamental e características do trabalho da população. 
A média de AF relatada pelos trabalhadores durante a jornada de trabalho foi de 3853,0 minutos por semana ( $\mathrm{min} / \mathrm{sem}$ ) (DP 583,4). A média de tempo despendido em atividades de deslocamento foi de 257,9 $\mathrm{min} / \mathrm{sem}$ (DP 237,5), durante o período de lazer foi de 77,6 min/sem (DP 111,5) e em atividades no domínio doméstico foi de 103,5 min/sem (DP 101,8). O escore médio de AF total relatado pelos participantes foi de $4292,0 \mathrm{~min} / \mathrm{sem}$ (DP 1034,2) (Tabela II).

Tabela 2 - Nível de AF (médias e medianas) nos diferentes domínios dos coletores de lixo de Pelotas e Rio Grande/RS, 2012 ( $N=127)$.

\begin{tabular}{lccccc}
\hline DOMínIO & $\begin{array}{c}\text { MÉDIA } \\
(\mathrm{min} / \mathrm{sem})\end{array}$ & $\begin{array}{c}\text { DESVIO-PADRÃO } \\
(\mathrm{min} / \mathrm{sem})\end{array}$ & $\begin{array}{c}\text { MEDIANA } \\
(\mathrm{min} / \mathrm{sem})\end{array}$ & $\begin{array}{c}\text { MÍNIMO } \\
(\mathrm{min} / \mathrm{sem})\end{array}$ & $\begin{array}{c}\text { MÁXIMO } \\
(\mathrm{min} / \mathrm{sem})\end{array}$ \\
\hline Trabalho & 3853,0 & 583,4 & 3840,0 & 1440,0 & 5400,0 \\
\hline Deslocamento & 257,9 & 237,5 & 210,0 & 0,0 & 900,0 \\
\hline Lazer & 77,6 & 111,5 & 60,0 & 0,0 & 720,0 \\
\hline Doméstica & 103,5 & 101,8 & 70,0 & 0,0 & 500,0 \\
\hline TOTAL & 4292,0 & 1034,2 & 4180,0 & 1440,0 & 7520,0 \\
\hline
\end{tabular}

Min/sem $=$ minutos por semana

Os coletores de lixo relataram a realização de níveis elevados de AF, observando-se escores mais altos durante o trabalho em comparação aos outros três domínios. Em média, o domínio ocupacional apresentou $89,8 \%$ do total de minutos de AF realizada (Figura I). Dentre as atividades praticadas durante o trabalho, a média de caminhada foi de $143 \mathrm{~min} / \mathrm{sem}$ (DP 171), em AF vigorosas, foi de 1313 $\mathrm{min} / \mathrm{sem}$ (DP 442) e em AF moderadas foi de $1083 \mathrm{~min} / \mathrm{sem}$ (DP 465).

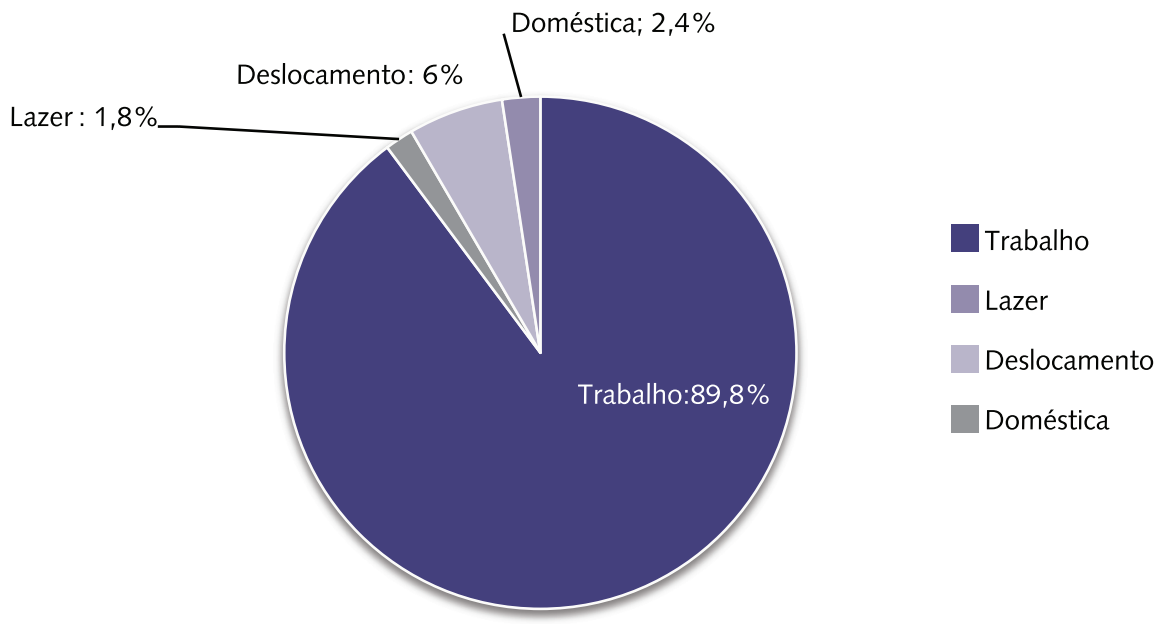

Figura 1 - Percentual (\%) de atividade física praticada nos diferentes domínios dos coletores de lixo de Pelotas e Rio Grande/RS, 2012 (N=127).

Os trabalhadores também apresentaram altos níveis de AF durante o deslocamento. Dentre os coletores de lixo, 61,4\% realizam deslocamento ativo e, destes, 61\% relataram se deslocar de bicicleta, em média por $211 \mathrm{~min} / \mathrm{sem}$ (DP238), e 39\% através da caminhada, por $46 \mathrm{~min} / \mathrm{sem}$ em média (DP 123).

Os menores índices de AF foram relatados no domínio do lazer. Dentre as atividades realizadas, a caminhada foi relatada por $5 \%$ dos participantes; $24 \%$ relataram praticar $\mathrm{AF}$ vigorosas e; $18 \%$ afirmaram realizar $\mathrm{AF}$ moderadas.

A maioria dos coletores de lixo (78\%) relatou realizar também AF no ambiente doméstico. As AF moderadas realizadas no pátio ou jardim de suas casas foram as mais relatadas. 


\section{DISCUSSÃO}

A média de AF total relatada pelos trabalhadores no presente estudo foi de 4292,0 minutos por semana (DP 1034,2) e, quando medida a AF no contexto ocupacional, o escore médio relatado foi de 3853,0 minutos por semana (DP 583,4). Nos domínios de deslocamento, lazer e doméstico, as médias de AF semanal foram, respectivamente, de 257,9 (DP 237,5), 77,6 (DP 111,5) e 103,5 (DP 101,8) minutos por semana.

A revisão de literatura realizada encontrou poucos estudos conduzidos no Brasil abordando a população de coletores de lixo. A maioria destes estudos abordou o processo e acidentes de trabalho ${ }^{2,4}$; outros descreveram a percepção dos indivíduos sobre o seu trabalho ${ }^{4,11}$ e averiguaram e discutiram a exigência física no trabalho a partir da frequência cardíaca ${ }^{5,6}$. Outros enfoques de estudo foram saúde psicológi$\mathrm{ca}^{12}$ e consumo de bebidas alcoólicas ${ }^{13}$. Nenhum dos estudos procurou determinar o nível de AF destes profissionais (principal exigência para cumprir a tarefa laboral), os quais realizavam tarefas que demandam grande exigência física de suma importância na preservação ambiental e para a saúde pública.

Com relação às características sociodemográficas, os coletores de lixo estudados apresentaram uma média de idade (26,2 anos) um pouco abaixo da apresentada pela literatura ${ }^{5,14}$. A baixa idade média verificada provavelmente ocorreu em função da elevada exigência física do trabalho executado ${ }^{4,5}$.

Ficou clara, também, uma grande rotatividade no trabalho (mediana de tempo de trabalho de 8,5 meses), 56\% dos indivíduos estavam a menos de um ano na função de coletor de lixo, assim como encontrado por Krajewski et al. ${ }^{15}$; sendo que $17 \%$ estavam a menos de um mês. Esse achado pode ser explicado pela forma de contrato realizado, o qual era renovado a cada três meses, dependendo do interesse do trabalhador e da empresa. A outra hipótese é a alta exigência da atividade, tanto física quanto psicológica, a qual pode levar a uma desistência do trabalho após períodos curtos de tempo.

Quanto aos hábitos comportamentais, os dados de tabagismo encontrados (34 $\%)$, foram muito semelhantes àqueles relatados por Mehrdad et al. (32\%) em seu estudo com coletores de lixo ${ }^{14}$; e mais que o dobro que a apresentada pela população brasileira $(15,1 \%)^{17}$. Este achado pode ser explicado pelo fato de que a prevalência de tabagismo em trabalhadores no Brasil é maior naqueles indivíduos que ocupam cargos com exigência de menor nível de escolaridade e maior esforço braça ${ }^{16}$. Já a prevalência de ingestão de bebidas alcoólicas apresentada $(57,9 \%)$ foi inferior ao encontrado por Mabuchi et al., os quais relataram frequências superiores a $90 \%{ }^{13}$. Os autores salientam que não se pode afirmar que o tipo de trabalho justifica tal comportamento já que é influenciado por diversos fatores.

A média de IMC apresentada pelos coletores $\left(23,4 \mathrm{~kg} / \mathrm{m}^{2}\right)$ é semelhante à encontrada por Anjos et al., $\left(22,9 \mathrm{~kg} / \mathrm{m}^{2}\right)$ em coletores de lixo da cidade do Rio de Janeiro $^{5}$. Esses dados podem ser reflexo da alta demanda física no trabalho, como também devido ao fato de que a atividade seja específica à este tipo físico; logo os indivíduos já ingressam na empresa com estas características. No presente estudo, apenas dois indivíduos foram classificados como obesos pelo IMC, e ambos se encontravam de licença médica, consequência de acidentes no trabalho.

A literatura apresenta um grande número de estudos focados em $\mathrm{AF}$ e os mais diversos fatores associados. No Brasil, o aumento na realização destes trabalhos se deu a partir do final do século XX, coincidentemente junto à inserção da educação física com a área da saúde ${ }^{17}$. No entanto, a análise dos domínios da $\mathrm{AF}$ estudados 
chama a atenção. Em revisão de literatura, Hallal et al. ${ }^{17}$ concluíram que o lazer é o domínio mais investigado no Brasil. Os autores reportaram que enquanto 20 artigos avaliaram o domínio do lazer, nove avaliaram a prática de AF total e nenhum estudo avaliou somente o domínio ocupacional ${ }^{17}$. Outro ponto que chama atenção é a inexistência de estudos avaliando o nível de AF de coletores de lixo no Brasil. Uma revisão de literatura realizada por Azevedo et al., incluiu 27 estudos referentes as mais diversas profissões e não encontrou estudos com a população em questão ${ }^{18}$.

Todavia, o domínio ocupacional deve ser considerado na realização de estudos no Brasil, onde, diferentemente de países desenvolvidos, a AF realizada no deslocamento para o trabalho, no próprio trabalho e nos serviços domésticos são muito frequentes $^{19}$. Além disto, alguns estudos tem demonstrado que estes domínios estão associados à redução do risco de morte prematura por doenças crônicas não transmissíveis ${ }^{20}$.

Muitas podem ser as justificativas para que o domínio ocupacional seja menos estudado, destacando-se entre elas a maior dificuldade de mensuração quando comparado aos domínios de lazer e deslocamento. Estudo conduzido no Brasil e na Colômbia identificou algumas limitações apresentadas pelo IPAQ especialmente na mensuração de AF no trabalho e nas tarefas domésticas ${ }^{21}$. Os entrevistados apresentam dificuldade de compreensão sobre as frações de tempo de 10 minutos e com relação à intensidade das atividades e tendem a exagerar o relato da $\mathrm{AF}^{21}$. No entanto, devido à alta exigência física da atividade ocupacional dos coletores de lixo, bem como da forma como o questionário foi aplicado, por entrevistadores bem treinados com a utilização de exemplos, acreditamos que os dados coletados refletem a realidade da $\mathrm{AF}$ realizada por estes trabalhadores.

Os escores de AF no domínio do trabalho encontrados neste estudo são diferentes dos resultados apresentados pela grande maioria das profissões ${ }^{22,23} \mathrm{e}$ da população ${ }^{24}$. Estudo com profissionais de academias de ginástica ${ }^{23}$ na cidade de Pelotas/RS, mostrou mediana de prática de AF no domínio de trabalho de 600 $\mathrm{min} / \mathrm{sem}$. Pitanga e colaboradores ${ }^{24}$ relataram média de AF ocupacional de aproximadamente $400 \mathrm{~min} / \mathrm{sem}$ em indivíduos do sexo masculino da população acima de 18 anos de um município do interior da Bahia. O presente estudo relatou média e mediana de AF laboral dez vezes superior aos escores encontrados na população em geral ${ }^{24} \mathrm{e}$ em uma população considerada muito ativa no trabalho ${ }^{23}$. A coleta de lixo apresenta uma exigência física significativamente alta, a qual envolve caminhadas, corridas e levantamento de pesos variados ao longo do dia ${ }^{5}$, especialmente em cidades onde a coleta ainda é realizada prioritariamente de forma manual, como é o caso dos municípios estudados. Desta forma, podemos afirmar que estes trabalhadores estavam constantemente sujeitos a riscos físicos e ergonômicos ${ }^{4}$, o que pode aumentar o risco do desenvolvimento de agravos osteomusculares.

Os coletores de lixo apresentaram também altos níveis de AF durante o deslocamento. Este domínio também mostrou resultado contraditório em relação à literatura, a qual identifica alta prevalência de inatividade física ${ }^{22,25}$. Um estudo realizado com trabalhadores da indústria do estado do Rio Grande do Sul, Brasil, encontrou que apenas $26,5 \%$ dos participantes caminhavam ou andavam de bicicleta para ir ao trabalho ${ }^{25}$. No entanto, encontramos que, além da alta demanda de trabalho, os coletores se utilizavam de bicicleta e caminhada para ir à empresa e, por morarem longe, gastavam bastante tempo no deslocamento. Dados de estudo demonstram uma relação inversa entre renda e nível de $\mathrm{AF}$ no deslocamento ${ }^{26}$, o que pode ajudar a explicar os resultados encontrados. Outro fator que pode contribuir para este achado é a geografia das cidades, as quais são predominantemente planas, o que facilitou o deslocamento ativo. 
Os menores índices de AF foram relatados no domínio do lazer. Tal resultado segue a tendência encontrada entre os indivíduos do sexo masculino moradores nas capitais dos 26 estados brasileiros e $\mathrm{DF}^{27}$ e de estudo realizados com grupos de trabalhadores ${ }^{28}$. Silva et al. ${ }^{28}$, realizaram um estudo com catadores de lixo da cidade de Pelotas/RS e encontraram prevalência de AF suficiente no lazer de 29,7\% entre os participantes. Dentre as barreiras à $\mathrm{AF}$ neste domínio mais relatadas por trabalhadores, está o cansaço ${ }^{29}$. O cansaço, oriundo da grande demanda física exigida pela coleta de lixo, é a provável explicação para o resultado encontrado neste estudo. Outro fator que pode contribuir é a carga horária de trabalho, que alcançou à marca das 14 horas em determinados dias.

O presente estudo apresentou um cuidado metodológico que deve ser ressaltado, especialmente no que se refere à elaboração do questionário. Além disto, a questão logística e condução das entrevistas, as quais contribuíram para a inexistência de perdas e recusas, são também pontos a serem destacados. Por outro lado, é sabido que as melhores estimativas de AF são obtidas através de medidas objetivas e que o relato através de questionários pode incorrer na superestimação do nível de AF. No entanto, o fato da coleta ter sido realizada por entrevista face -a-face, reconhecidamente diminui a ocorrência desse erro $^{30}$.

Tendo em vista os achados do estudo, conclui-se que os coletores de lixo estavam expostos a uma carga extremamente elevada de AF, especialmente no domínio do trabalho, podendo ocasionar prejuízo significativo para a saúde se não forem adotadas medidas adequadas de ergonomia, nutrição e recuperação física.

Assim, sugere-se que as empresas responsáveis por esse tipo de serviço atentem para a carga horário de seus trabalhadores, fornecendo pausas adequadas durante a jornada de trabalho, de maneira a permitirem uma compensação da demanda fisiológica de seus trabalhadores. Além disto, forneçam uma alimentação balanceada aos mesmos antes, durante e após sua jornada de trabalho, de forma a suprir sua necessidade energética.

\section{Contribuição dos autores}

Rodrigo Kohn Cardoso coordenou o trabalho de campo, conduziu a revisão de literatura e realizou a escrita e criação de tabelas e figuras do artigo. Marcelo Cozzensa da Silva idealizou o estudo, conduziu a análise de dados e revisão crítica da escrita do artigo. Aírton José Rombaldi participou da escrita e análise de dados do artigo.

\section{Agradecimentos}

À direção da empresa Revita, responsável pela coleta de lixo nas cidades de Rio Grande e Pelotas. A todos os coletores de lixo que ajudaram na realização deste estudo.

\section{REFERÊNCIAS}

1. Associação Brasileira das Empresas de Limpeza Pública e Resíduos Especiais - ABRELPE. Panorama dos Resíduos Sólidos no Brasil 2010. [citado 2012 jul 20] Disponível em: http:// www.abrelpe.org.br/panorama_edicoes.cfm.

2. Ferreira J, Anjos L. Aspectos de saúde coletiva e ocupacional associados à gestão de resíduos sólidos municipais. Cad Saúde Pública. 2001;17:689-96.

3. EnglehardtJD, Fleming LE, Bean JA. Analytical predictive Bayesian assessment of occupational injury risk: municipal solid waste collectors. Risk Anal. 2003; 23(5):917-27.

4. Velloso MP. Processo de trabalho e acidentes de trabalho em coletores de lixo domiciliar na cidade do Rio de Janeiro, Brasil. Cad Saúde Pública. 1997; 4(13):693-700. 
5. Anjos LA, Ferreira JA, Damião IJ.Heart rate and energy expenditure during garbage collection in Rio de Janeiro, Brazil. Cad Saúde Pública. 2007; 23(11): 2749-55.

6. Anjos LA, Ferreira JA. A avaliação da carga fisiológica de trabalho na legislação brasileira deve ser revista! O caso da coleta de lixo domiciliar no Rio de Janeiro. Cad Saúde Pública. 2000; 16(3):785-90.

7. World Health Organization - WHO. Physical activity. [citado 2012 fev 02] Disponível em: http://www.who.int/topics/physical_activity/en/.

8. Associação Brasileira de Empresas de Pesquisa - ABEP. Critério de classificação econômica do Brasil. [citado 2012 ago 10] Disponível em: http://www.abep.org/novo/Content. aspx?SectionID=84.

9. Rounsaville BJ, PolingJ.Substance use disorders measures.In:American Psychiatric Association. Handbook of psychiatric measures. Washington: American Psychological Association; 2000. p. 457-84.

10. Craig CL, Marshall AL, Sjostrom M. International physical activity questionnaire: 12 -country reliability and validity. Med Sci Sports Exerc. 2003; 35(8):1381-95.

11. Lazzari MA, Reis CB. The perception of urban garbage collectors of Dourados, in the state of Mato Grosso do Sul, regarding the biological risks involved in their work routine. Cien Saúde Colet. 2011; 16(8):3437-42.

12. Barbosa SC, Melo SL, Medeiros UM, Vasconcelos, T. M. Perfil de bem-estar psicológico em profissionais de limpeza urbana. Revista Psicologia: Organizações e Trabalho 2010; 10(2):54-66.

13. Mabuchi AS, Oliveira DF, Lima MP, Conceição MB, Fernandes H. The use of alcohol by workers of the garbage collection service. Rev Latino-Am Enfermagem 2007; 15(3):446-52.

14. Mehrdad R, Majlessi-nasr M, Aminian O, Sharifian S, Malekahmadi F. Musculoskletal disorders among solid waste workers. Acta Med Iran. 2008; 3(46): 233-38.

15. Krajewski JA, Tarkowski S, Cyprowski M, Szarapińska-kwaszewska J, Dudkiewicz B. Characteristics of jobs and workers employed in municipal waste collection and disposal by the city of Lodz. Int J Occup Med Environ Health. 2002; 15(3):289-301.

16. Barros AD, Cascaes AM, Wehrmeister FC, Martínez-mesa J, Menezes AB. Tabagismo no Brasil: desigualdades regionais e prevalência segundo características ocupacionais. Cien Saúde Colet. 2011; 16(9):3707-16.

17. Hallal PC, Dumith SC, Bastos JP, Reichert FF, Siqueira FV, Azevedo MR. Evolution of the epidemiological research on physical activity in Brazil: a systematic review. Rev Saúde Pública. 2007; 41(3):453-60.

18. Azevedo SF, Lopes AS. Atividade física desempenhada por trabalhadores brasileiros: uma revisão sistemática. Pensar a Prática 2012; 15(3).

19. Martinez-Gonzalez MA, Varo IJ, Santos JL, Irala J, Gibney MJ, Kearney J, et al. Prevalence of physical activity during leisure time in the European Union. Med Sci Sports Exerc. 2001; 33(7):1142-6.

20. Arrieta A, Russell LB. Effects of leisure and non-leisure physical activity on mortality in U.S. adults over two decades. Ann Epidemiol. 2008; 18:889-95.

21. Hallal PC, Gomez LF, Parra DC, Lobelo F, Mosquera J, Florindo A, et al. Lessons learned after 10 years of IPAQ use in Brazil and Colombia. J Phys Act Health. 2010; 7(2): 259-64.

22. Abu-omar K, Rutten A. Relation of leisure time, occupational, domestic, and commuting physical activity to health indicators in Europe. Prev Med. 2008; 47: 319-23.

23. Hartwig TW, Silva MC, Reichert FF, Rombaldi AJ. Condições de saúde de trabalhadores de academias da cidade de Pelotas/RS: um estudo de base populacional. Rev Bras Ativ Fis Saude. 2012; 17(6): 500-11.

24. Pitanga FJG, Almeida LAB, Freitas MM, Pitanga CPS, Beck CC. Padrões de atividade física em diferentes domínios e ausência de diabetes em adultos. Motricidade. 2010; 6(1): 5-17.

25. Silva SG, Del duca GF, Silva KS, Oliveira ES, Nahas MV. Commuting to and from work and factors associated among industrial workers from Southern Brazil. Rev Saúde Pública. 2012; 46(1):180-4.

26. Del Duca GF, Rombaldi AJ, Knuth AG, Azevedo MR, Nahas MV, Hallal PC. Associação entre nível econômico e inatividade em diferentes domínios. Rev Bras Ativ Fís Saúde. 2009; 14(2):123-31. 
27. Malta DC, Moura EC, Castro AM, Cruz DA, Neto OM, Monteiro CA. Padrão de atividade física em adultos brasileiros: resultados de um inquérito por entrevistas telefônicas, 2006. Epidemiol Serv Saúde 2009; 18(1):7-16.

28. Silva M, Fassa A, Kriebel D. Leisure time physical inactivity among Brazilian ragpickers. Rev Bras Ativ Fís Saúde. 2011;16(2):125-31.

29. Silva SG, Silva MC, Nahas MV, Viana SL. Fatores associados à inatividades física no lazer e principais barreiras na percepção de trabalhadores da indústria do sul do Brasil. Cad Saúde Pública. 2011; 27(2): 249-59.

30. Freitas MP, Silva MC, Bandeira FM, Hallal PC, Rombaldi AJ. Associação entre aptidão cardiorrespiratória e nível de atividade física em adultos jovens. Rev Bras Ativ Fís Saúde. 2013;18(2):260-70.

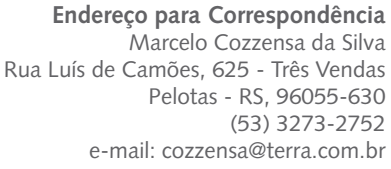

Recebido 28/10/2013

Revisado 21/11/2013

Aprovado 22/11/2013 